\title{
Audit of perioperative pain management in paediatric patients following tonsillectomy at a tertiary hospital in Johannesburg
}

\author{
Mogane PN* (D, Mashinini M, Lundgren C \\ Department of Anaesthesiology, School of Clinical Medicine, Faculty of Health Sciences, University of the Witwatersrand \\ *Corresponding author, email: moganep@gmail.com
}

Background: Adenotonsillectomy remains one of the most frequently performed surgical procedures in children. Despite improvements in anaesthetic and surgical techniques, severe pain is reported in as many as $25-50 \%$ of children. Pain assessment and knowledge of drug pharmacodynamics and pharmacokinetics in the paediatric patient, is a prerequisite for optimal care. Much has been written on perioperative pain management following tonsillectomy. However, no consensus has been reached on what the ideal analgesic regimen should be.

This audit is a review of current practice at Chris Hani Baragwanath Academic Hospital. It aims to identify problems and develop possible solutions to improve anaesthetic practice.

Methods: A prospective, contextual, descriptive study design using a data collection sheet was used on paediatric patients presenting for tonsillectomy.

Results: Eighty-five patients aged three to 12 years of age, with ASA grading I or II, were enrolled in the study. The choice of anaesthetic was variable with a combination of simple analgesics, opioids and adjuvants. This affected postoperative pain scores. Snare dissection and monopolar cautery haemostasis, was the standard surgical technique. Surgical seniority influenced the duration of tonsillectomy, with an effect on postoperative pain scores.

Conclusions: Audits are necessary to evaluate what resources are needed to optimise care. The occurrence of pain after tonsillectomy continues to be poorly managed. Appropriate premedication and fasting of clear fluids for no more than two hours, needs to be introduced. Where possible surgical technique should involve bipolar cautery and be limited to less than 45 minutes. A preemptive, multimodal, opioid-sparing anaesthetic should be routinely practised.

Keywords: anaesthesia, paediatric, tonsillectomy, pain management, audit

\section{Introduction}

Adenotonsillectomy remains one of the most frequently performed surgical procedures in children. It presents risks and challenges for both surgeon and anaesthetist. ${ }^{1-3}$ Despite improvements in anaesthetic and surgical technique, poor pain control, postoperative nausea and vomiting (PONV), and emergence delirium continue to be common complications. In fact, severe pain is reported in as many as $25-50 \%$ of children presenting for adenotonsillectomy. ${ }^{4}$ Acute pain is defined as a "normal, predicted, unpleasant sensory and emotional response to an adverse chemical, thermal or mechanical stimulus" 5,6 This definition recognises both the physiological and affective nature of the pain.

Multiple factors contribute to the experience of pain. A systematic review published in 2016 titled "Predisposing, precipitating, perpetuating and present factors predicting anticipatory distress to painful medical procedures in children" looked at this. Of note is the high positive relationship between anticipatory anxiety and the level of postoperative pain, the role that parental anxiety plays, and the role that idiosyncratic needs such as fatigue, hunger, dehydration and nausea have on children's anticipatory distress.? Surgical techniques also contribute to pain. Often the choice will be based on surgeon preference, experience and instrumentation available. Cold dissection (scalpel, guillotine and snare) and hot dissection (monopolar and bipolar electrocautery) are commonly described. ${ }^{8,9}$ Coblation, laser and the microdebrider are less frequently used. All these techniques are associated with variable postoperative pain intensities.

Pain assessment is imperative for optimal pain management. Without appropriate methods of assessment, it is difficult to plan adequate interventions and to take the necessary steps to ensure their effectiveness. One suggested approach is that of QUESTT ${ }^{10}$ :

- "Question the child

- Use the age and developmentally appropriate pain-rating scales

- Evaluate behaviour and physiological changes

- Secure parental involvement

- Take the cause of pain into account

- Take action and evaluate results"

Most of the pain-rating scales are not validated, applicable to all age groups or universally accepted. In general it is recognised that there are physiological measures, behavioural measures and self-report. ${ }^{10-12}$ Self-report is often referred to as the 'gold standard' as it is a direct measure of pain. The self-report tool used must be appropriate to the child's age and developmental level; it must be practical to use in the clinical setting; it should be reproducible, reliable and valid; and ideally transferable between assessors. These tools can be used in children as young as three years of age. ${ }^{10}$ However, one must be aware that they may be 
subject to individual response biases, reflecting the child's interpretation of the pain report, ${ }^{13}$ thus resulting in misleading scores. For this reason it is advised that self-report scales be corroborated with observational measures in children younger than five years of age.

Anaesthetic techniques that contribute to perioperative pain are difficult to isolate. The use of suitable premedication has been found to decrease anticipatory anxiety associated with surgery, and potentially contributes to the decrease in occurrence of early postoperative negative behaviour i.e. emergence delirium and pain. ${ }^{14,15}$ Pain management varies markedly depending on practitioner preference, resources, surgeon, and patient population. It is difficult to apply a "one size fits all" recommendation, and the efficacy of any intervention must be assessed and titrated in individual patients. A large number of otherwise healthy children undergo tonsillectomies which are often associated with significant pain. This provides a useful model for the study of perioperative pain in the paediatric patient. Findings from this population can be used to assess the system for other surgical procedures.

An audit is part of a cycle of quality assurance. It is a review of current practice, identification of problems, and the development of possible solutions with the aim of implementation and improvement in response to the analysis. ${ }^{16}$ This study aimed to examine current strategies employed in patients presenting for adenotonsillectomy, by reviewing anaesthetic and surgical techniques and prescriptions, and their contribution to postoperative pain.

\section{Method}

Ethical approval for this study was sought from the Human Research Ethics Committee (Medical) of the University of the Witwatersrand. Approval for conducting the study was obtained from the Postgraduate committee, and the Chief Executive Officer (CEO) of Chris Hani Baragwanath Academic Hospital (CHBAH). A prospective, contextual, descriptive research design was used. A comprehensive information letter and clear explanation of the study was given to the parent or caregiver of all participants; signed informed consent was mandatory prior to inclusion in the study. In-hospital data was collected from the anaesthetic and surgical records. All information was captured anonymously in a data collection form. A summary of the questionnaire is shown in Table I.

The following patients were excluded from the study:
- Emergency surgery

- ASA III to V, presence of confirmed genetic or metabolic syndrome

- Children with behavioural disorders or cognitive impairment

- A history of chronic pain or preoperative use of analgesic drugs

- Any patients requiring preoperative sedation or total intravenous anaesthesia

- Any patient in whom consent could not be obtained

- Participants in whom it was not possible to use the WongBaker pain scale

In consultation with a biostatician, an appropriate sample size was calculated at $62-82$ patients. The sample size estimation was based on one of the key research questions which was the identification of the presence of pain (Wong Baker score $\geq 4 / 10$ ).

Data was captured onto a Microsoft Excel ${ }^{\circ} 2010$ spreadsheet and analysed using SAS (version 9.4 for Windows). Descriptive analysis of the data was carried out as follows:

- Categorical variables were summarised by frequency and percentage tabulation, and illustrated by means of bar charts.

- Continuous variables were summarised by median and interquartile range, and their distribution illustrated by means of histograms.

- For tests of association, the pain scores were categorised as 0-2 (none/mild), 4-6 (moderate), 8-10 (severe).

The $\mathrm{X}^{2}$ test was used to assess the relationship between categorised pain score and categorical variables. Fisher's exact test was used for $2 \times 2$ tables or where the requirements for the $\mathrm{X}^{2}$ test could not be met. The strength of the associations was measured by Cramer's V and the phi coefficient respectively. The following scale of interpretation was used:

0.50 and above

high/strong association

0.30 to 0.49

moderate association

0.10 to 0.29

weak association

below 0.10

little if any association

The relationship between categorised pain score and continuous variables was assessed by the t-test (or ANOVA test for more than two categories). Where the data did not meet the assumptions of these tests, a non-parametric alternative, the Wilcoxon rank sum test (or the Kruskal-Wallis test for more than two categories) was used. The strength of the associations was measured by

Table I. Summary of the questionnaire (data collection form)

\begin{tabular}{lll}
\hline Section & Intention & Description \\
\hline Patient information & Demographic characteristics & Age, gender, weight, ASA rating and fasting duration \\
Intraoperative information & $\begin{array}{l}\text { Anaesthetic and surgical } \\
\text { information }\end{array}$ & Anaesthetic and surgical expertise, times, techniques and complications \\
Postoperative information & Pain scores and complications & $\begin{array}{l}\text { Perioperative pain rating: preoperative, at first verbalisation, } 30 \text { minutes and } \\
\text { three hours postoperative, and at discharge; postoperative prescription; } \\
\text { complications and parental satisfaction }\end{array}$ \\
\hline
\end{tabular}


Table II. Demographics, fasting, surgical and anaesthetic duration

\begin{tabular}{lcccccc}
\hline Variable & N & Median & Interquartile range & Minimum & Maximum \\
\hline Age $(\mathrm{y})$ & 85 & 5 & 4 & 7 & 3 & 11 \\
Weight $(\mathrm{kg})$ & 85 & 18.3 & 15.6 & 23.0 & 10.4 & 46.0 \\
Fasting duration $(\mathrm{h})$ & 85 & 13.2 & 11.9 & 14.4 & 10.3 & 18.5 \\
Surgery duration $(\mathrm{min})$ & 85 & 20 & 15 & 35 & 5 & 125 \\
Anaesthesia duration $(\mathrm{min})$ & 85 & 45 & 35 & 60 & 15 & 146 \\
\hline
\end{tabular}

the Cohen's $d$ for parametric tests and the r-value for the nonparametric tests. The following scale of interpretation was used:

0.80 and above

0.50 to 0.79

0.20 to 0.49

below 0.20

The $5 \%$ significance level was used i.e. p-values $<0.05$ indicate significant associations.

\section{Results}

Ninety-seven patients aged three to 12 years of age, with ASA grading I (84\%) or II, who underwent an elective adenotonsillectomy were enrolled in the study. Twelve children had to be excluded due to incomplete data collection sheets. Fifty one percent of the patients were female. Demographic data, fasting period and duration of surgery and anaesthesia is displayed in Table II.

The majority of the procedures were performed in the presence of an anaesthetic consultant/principal medical officer (PMO) (55\%), while $37 \%$ and $8 \%$ were performed by senior and junior registrars respectively. Five percent $(n=4)$ of the population received premedication in the form of paracetamol $(n=1)$,

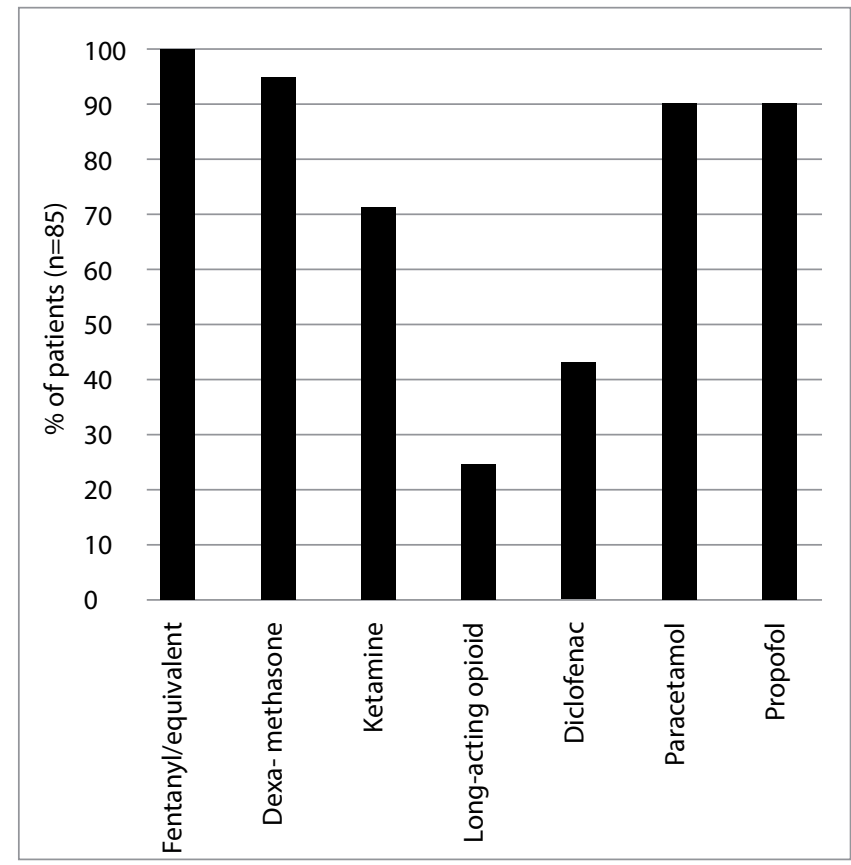

Figure 1. Intraoperative medication given

Fentanyl/equivalent = fentanyl, sufentanyl or alfentanyl; Long-acting opioid = morphine or tramadol midazolam ( $n=1)$ and dexmedetomidine $(n=2)$. Fifty-two percent of the patients received intraoperative prophylactic antibiotics in the form of cephazolin or augmentin.

Medication given intraoperatively is documented in Figure 1. Note that $86 \%(n=66)$ of the patients received intravenous paracetamol, and the remainder by rectum $(n=11)$. The status of the remainder of the patients was unknown. Eleven percent $(n=9)$ had local anaesthetic infiltrated in the tonsillar bed by the surgeon.

Intraoperative complications occurred in $15 \%(n=13)$ of the population. This included issues pertaining to the airway (laryngospasm, bronchospasm, hypercarbia, need to reintubate), bleeding and injury to tongue and teeth. Eighty-six percent $(n=73)$ of the patients were extubated awake.

Tonsil size was predominantly grade II (41\%) and III (32\%), and surgical technique was routinely by cold steel dissection and haemostasis achieved with unipolar diathermy. The majority of the surgeries were performed by registrars (45\%) and consultants $(36 \%)$, while only $19 \%$ were performed by a medical officer.

The percentage of children who were prescribed postoperative medication, for the ward as per the surgeon's prescription, is shown in Figure 2.

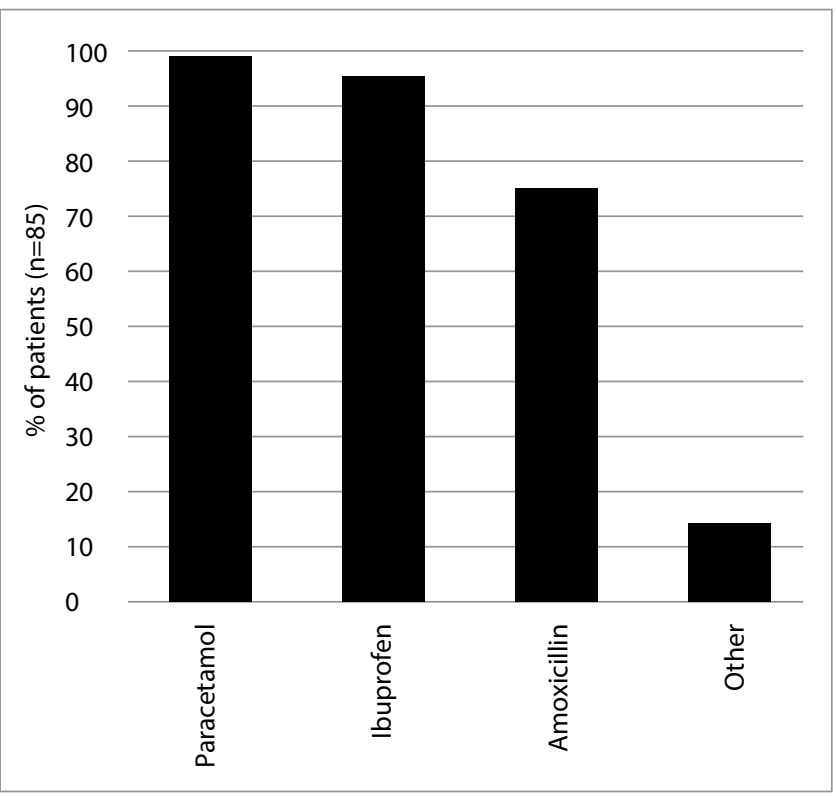

Figure 2. Postoperative medication

Other = bactroban ointment; ranitidine; beclometazone, fluticasone nasal spray; saline nose drops; prednisone; chlorpheniramine; glycothymol oral wash 


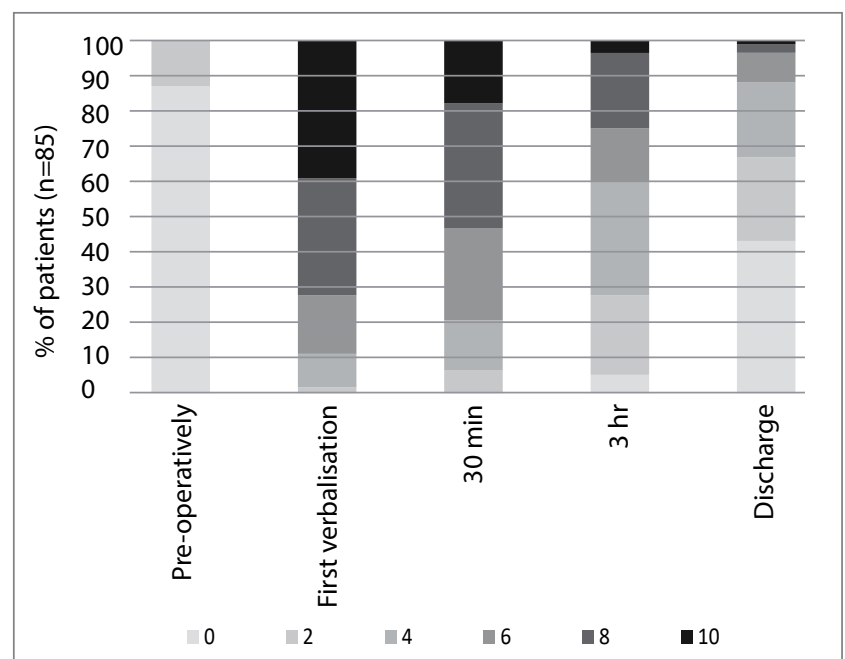

Figure 3. Pain profiles of patients across the five measurement occasions (presence of pain taken as Wong-Baker score $\geq 4 / 10$ )

Complications in the recovery room occurred in $97 \%(n=82)$ of the population. These included: pain $97 \%$; restlessness $32 \%$; PONV 5\%; airway problems in the form of stridor and snoring $5 \%$; and drowsiness $2 \%$. Eighteen of the 82 children who experienced pain, were given analgesics in the recovery room in the form of fentanyl, paracetamol or tilidine drops. The most common complication in the ward was ongoing pain (72\%). No rescue analgesia was given in the ward; instead it was given according to a combination of factors including: time of arrival from theatre; schedule as per nursing analgesic round; and ability to take orally.

The pain profile of the patients across the five measurement occasions is shown in Figure 3. The percentage of patients with severe pain (score 8 or 10) decreased from $72 \%$ at first verbalisation to $53 \%$ at 30 minutes, to $25 \%$ at three hours, to $4 \%$ at discharge.

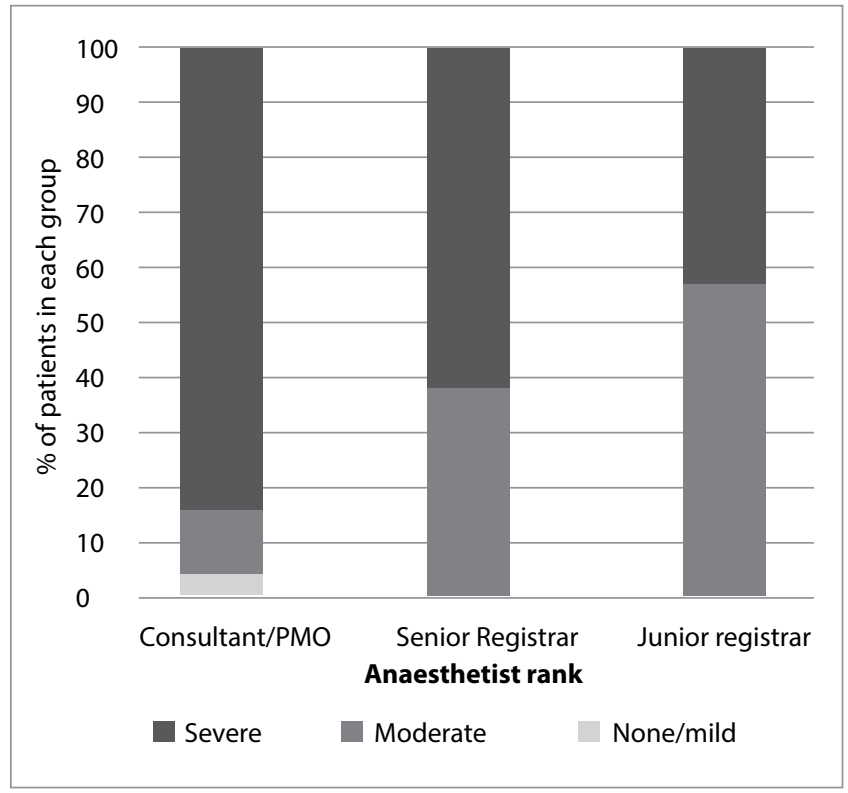

Figure 4. Comparison of categorised pain score at first verbalisation and anaesthetist rank

None $/$ mild pain $=$ Wong Baker score $\leq 2 / 10$; Moderate $=4-6 / 10$ and Severe $\geq 8 / 10$

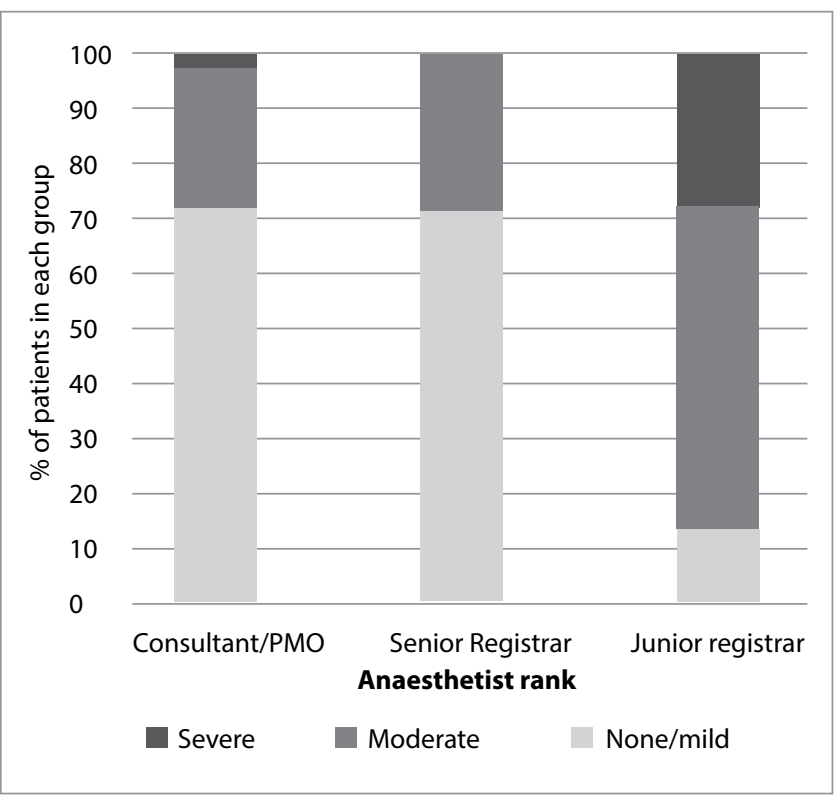

Figure 5. Comparison of categorised pain score at discharge from recovery and anaesthetist rank

There was a significant, moderate association between categorised pain score at first verbalisation and anaesthetist rank $(p=0.010$; phi coefficient $=0.37$ ). The percentage of patients with a severe pain score was higher for consultants/PMOs (83\%) compared to senior or junior registrars (61 and 43\% respectively) (Figure 4).

There was also a significant, moderate association between categorised pain score at discharge from recovery and anaesthetist rank ( $p=0.0050$; phi coefficient $=0.47$ ). The percentage of patients with a severe pain score was lower for consultants/PMOs and senior registrars ( 2 and $0 \%$ respectively) compared to junior registrars (29\%) (Figure 5).

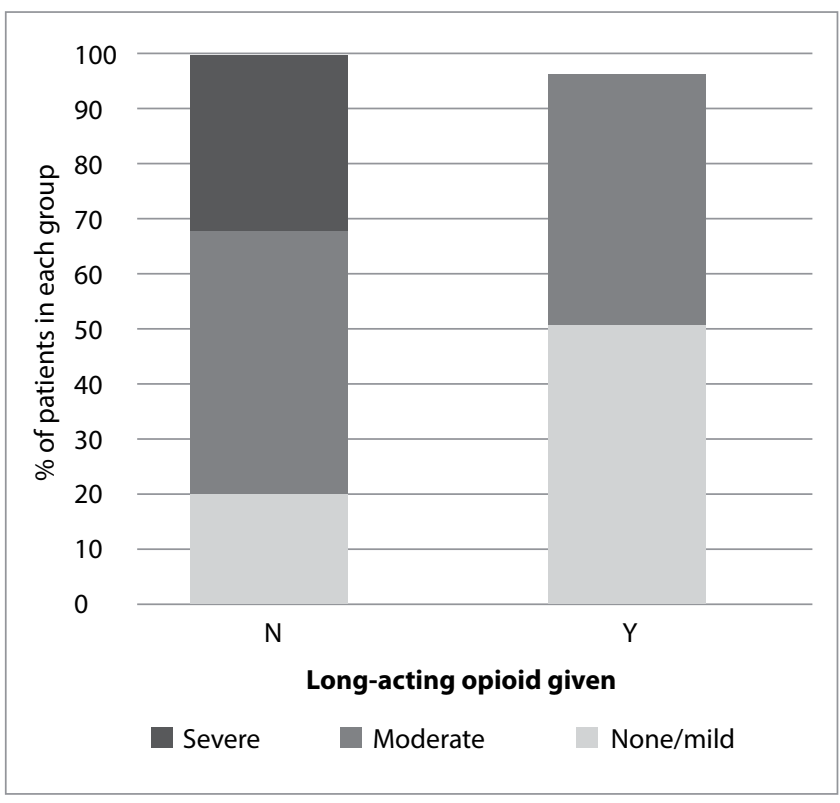

Figure 6. Comparison of categorised pain scores at $3 \mathrm{hrs}$ and usage of long-acting opioid

$\mathrm{N}=$ given fentanyl/equivalent only; $\mathrm{Y}=$ given fentanyl/equivalent and morphine or tramadol 


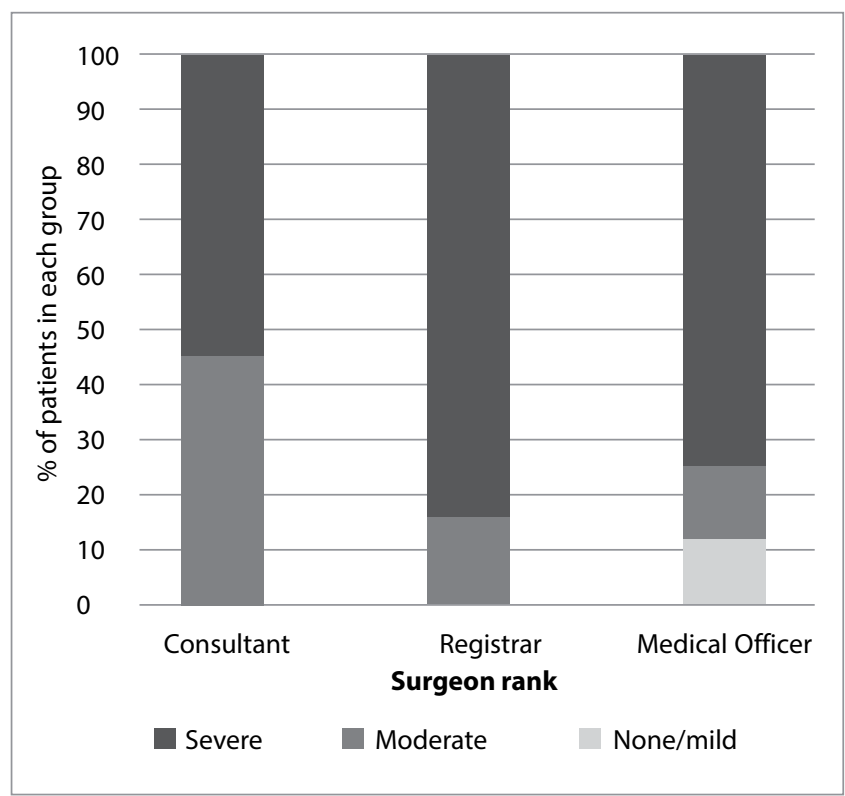

Figure 7. Comparison of categorised pain at first verbalisation and surgeon rank

There were no significant associations between anaesthetist rank and pain scores at the other two time points. There was a significant, moderate association between categorised pain score at three hours and whether or not a long-acting opioid was given ( $p=0.0072$; Cramer's $V=0.34$ ). The percentage of patients with a severe pain score was lower for those who had been given a long-acting opioid (5\%) compared to those who had not (32\%) (Figure 6). Long-acting opioid was prescribed more commonly (90\%) in consultant/PMO-lead cases, however, less than half (43\%) of the consultant/PMO-lead cases used longacting opioids.

There was a significant, moderate association between categorised pain score at first verbalisation and surgeon rank ( $p$ $=0.0027$; phi coefficient $=0.46$ ). The percentage of patients with a severe pain score was lower for consultants (55\%) compared to registrars or MOs (84 and 75\% respectively) (Figure 7). There were no significant associations between surgical rank and pain

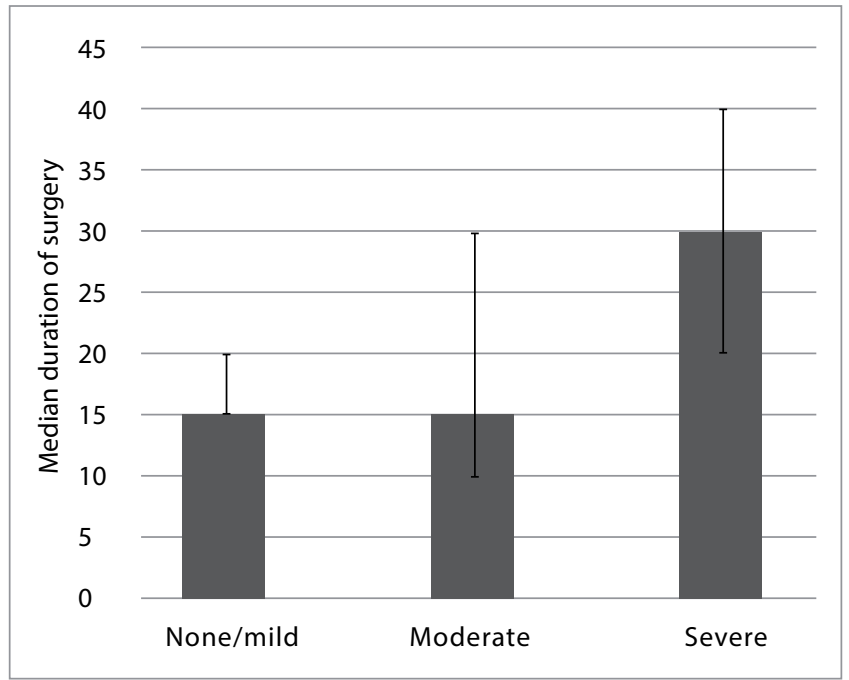

Figure 8. Comparison of median duration of surgery and categorised pain score at 30 minutes scores at other time points. Tonsil size was of no significance to pain scores at any time points.

A significant association was found between the median duration of surgery and categorised pain score at 30 minutes ( $p=0.0078$ ). Post-hoc tests showed that those with severe pain scores had longer median duration of surgery (30 min; IQR 20-40 $\mathrm{min}$ ) than those with moderate pain scores (15 min; IQR 10-30 min) or those with no/mild pain scores (15 min; IQR 15-20 $\mathrm{min}$ ). The effect sizes were moderate and small, respectively ( $r=$ 0.30 and 0.29 respectively) (Figure 8 ). There were no significant associations between duration of surgery and pain scores at other time points.

A significant association was found between the median duration of anaesthesia and anaesthetists rank ( $p<0.0001)$. Post-hoc tests showed that consultants/PMOs had longer median duration of anaesthesia (60 $\mathrm{min}$; IQR 45-70 $\mathrm{min}$ ) than the senior or junior registrars ( $35 \mathrm{~min}$; IQR $25-45 \mathrm{~min}$ and 30 min; IQR 30-60 min respectively) (Figure 9). The effect sizes were moderate ( $r=0.57$ and 0.33 respectively).

There was a significant association between the median duration of surgery and surgeon rank $(p<0.0001)$. Post-hoc tests showed that consultants had shorter median duration of surgery ( $15 \mathrm{~min}$;

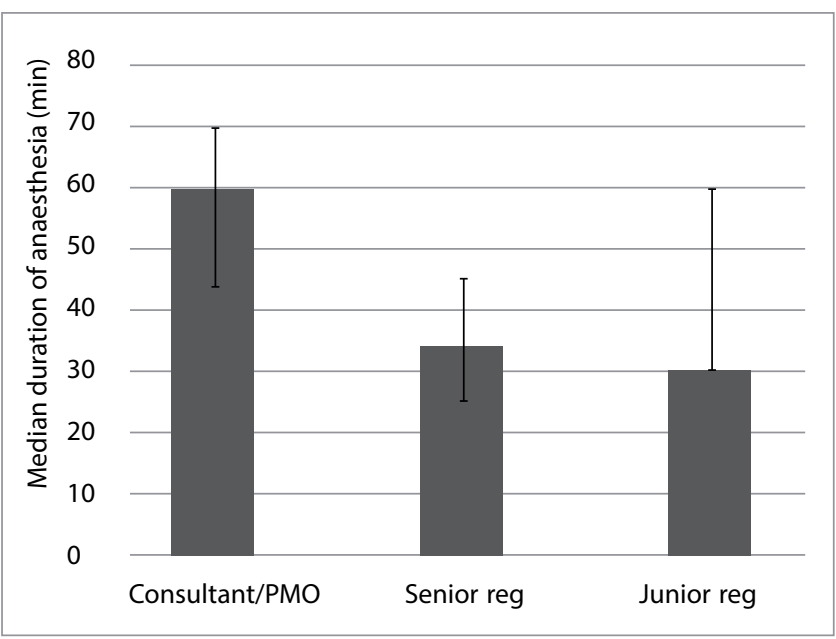

Figure 9. Comparison on duration of anaesthesia and anaesthetist rank

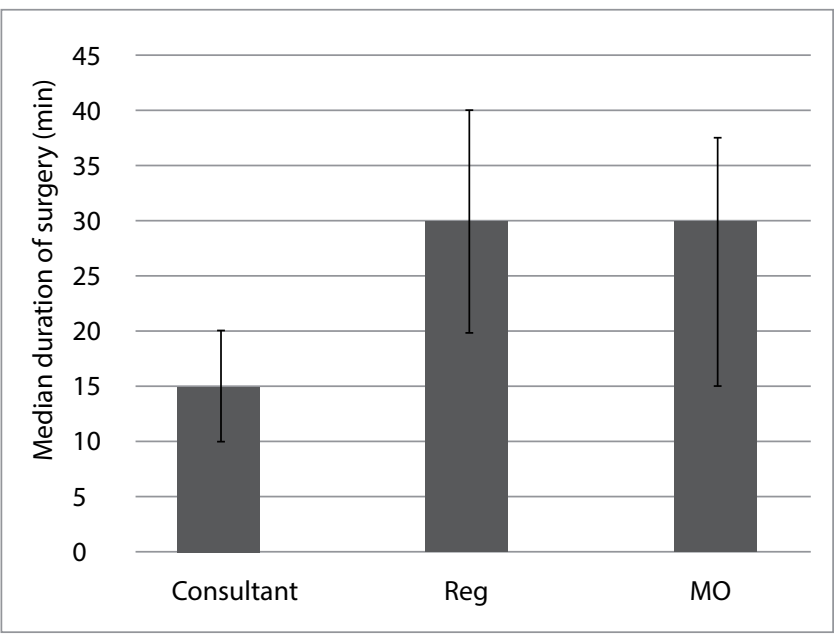

Figure 10. Comparison of duration of surgery and surgeon rank 
IQR 10-20 min) than the registrars or MOs (30 min; IQR 20-40 min and $30 \mathrm{~min}$; IQR 15-48 min respectively). The effect sizes were moderate $(r=0.60$ and 0.54 respectively) (Figure 10).

\section{Discussion}

Tonsillectomy is one of the commonest surgical procedures in children, ${ }^{1-4,8}$ yet it has been identified as a procedure associated with a high incidence of severe postoperative pain. Factors that contributed to this poorly managed pain in this audit included:

- Surgical technique and duration of surgery;

- Anaesthetic technique; inability to recognise and assess pain;

- Misconceptions/preconceived ideas about analgesic drugs;

- Child and system factors.

\section{Surgical technique}

Cold steel dissection (snare) and a variable degree of monopolar electrocautery to achieve haemostasis are used at CHBAH. Bipolar cautery should be used instead of monopolar electrocautery because it results in less tissue damage and therefore less postoperative pain. It also has the benefits of reduced intraoperative bleeding and surgical time. . $, 9,17^{2}$

In this study, surgical seniority was associated with shorter duration of surgery and lower incidences of severe pain at 30 minutes. Unfortunately, literature remains inconclusive regarding the difference of perioperative pain and varying degree of surgical experience. ${ }^{18,19}$ Anaesthetic seniority was associated with higher pain scores because consultants/PMO anaesthetists often operate with junior surgeons, while 'catchup' lists run by junior anaesthetists have consultant surgeons operating.

\section{Analgesia}

Evaluation of postoperative pain is complicated by the difficulty in assessing pain in children and the occurrence of emergence delirium. It is often difficult to differentiate between the pain and ED because of the overlapping clinical picture, and because pain can often be the cause of agitation. ${ }^{14}$ Furthermore, several studies have shown that otorhinolaryngological procedures, sevoflurane, and also preoperative anxiety, increase the risk of emergence delirium. ${ }^{14,15,20}$ To overcome this, pain assessment took place at multiple points and a self-report scale was used. The Wong-Baker scale, which was chosen, can be used in children as young as three, and is available in various local translations. ${ }^{21}$

In this audit, analgesic drugs used included short-acting opioids usually in the form of fentanyl (at a median dose of $1.8 \mathrm{ug} / \mathrm{kg})$; dexamethasone $(0.1 \mathrm{mg} / \mathrm{kg}$ ) and paracetamol (15 $\mathrm{mg} / \mathrm{kg}$ ). Lack of administration of these three was found to be due to unavailability. The use of NSAIDs perioperatively in adenotonsillectomy has been encouraged as they are opioidsparing, reduce PONV and time to first oral intake..$^{22,23}$ However, concerns seemed to exist about ketamine and long-acting opioids.
There is reluctance for practitioners to give long-acting opiates in children presenting for tonsillectomy. Only $25 \%$ of the study population received morphine or tramadol. This is due to fear of opioid-associated side-effects (e.g. sedation, respiratory depression, PONV, anoxia and death); pharyngeal surgery in children who are at a high risk of airway reactivity; and unpredictable postoperative monitoring. Interestingly, longacting opioids were commonly prescribed (90\%) in consultant/ PMO-lead cases resulting in improved pain scores at discharge from recovery (severe pain in $2 \%$ consultant lead vs $29 \%$ junior registrar lists). Three hours postoperative, severe pain was better in patients who had received long-acting opioids versus those who had not (5\% versus $32 \%$ respectively). This is due to individual drug pharmacokinetics. Literature on tramadol usage and adverse effects in children presenting for tonsillectomy is limited. Accurate risk-benefit analysis is thus not available, ${ }^{24}$ and until such time, the routine usage of tramadol cannot be recommended.

Recommendations are a multimodal combination of individually titrated intraoperative opioids (morphine $0.1 \mathrm{mg} /$ $\mathrm{kg}$ or tramadol $1 \mathrm{mg} / \mathrm{kg}$ to be considered), dexamethasone, and regularly-administered mild analgesics in the form of paracetamol and/or NSAIDs. Ketamine ( $\leq 0.5 \mathrm{mg} / \mathrm{kg}$ ) improves analgesia but its advantage must be carefully considered when administered with morphine. Dexmedetomidine ( $1 \mathrm{ug} / \mathrm{kg}$ ) or clonidine reduces opioid requirements and agitation especially in children with sleep disordered breathing, and is advised. ${ }^{13,25}$ Growing fears of opioids may eventually lead us to consider the usage of gabapentinoids, honey and non-pharmacological measures.

\section{Child and system factors}

Despite the stringent recommendations of the American Society of Anaesthetists, ${ }^{26}$ children in this study were routinely faced with 13.2 (minimum 10.3 and maximum 18.5) hours without eating or drinking. Appropriate fasting and adequate intraoperative hydration $(10-15 \mathrm{ml} / \mathrm{kg}$ ) has been shown to reduce the incidence of PONV, anxiety, agitation, dehydration, and in some studies has been associated with a decrease in pain. 27,28

Ninety-four percent of parents questioned were satisfied with the process, while six percent were not satisfied. Reasons given included: lack of communication, no knowledge of the process, prolonged theatre time without notification of wellbeing of child, long fasting times and pain postoperatively.

Shortage of staff and equipment contributes to several deficits in the delivery of both surgical and anaesthetic services at CHBAH. These 'system factors' can be improved by:

- Communication amongst all role players.

- Increase in functional equipment and monitoring would foster better premedicating and analgesic practices. 
- Shortening the hospital stay would have socio-economic benefits, and possibly encourage greater parental presence. Currently patients are admitted for $\geq 48$ hours.

\section{Limitations}

This study is contextual to Chris Hani Baragwanath Academic Hospital, and the results cannot be generalised to other hospitals in Johannesburg or in South Africa. A great deal of the practice is influenced by system errors which may not adhere to established norms. However, the findings in this study are in keeping with multiple studies in first world countries, and confirm the dilemma experienced in managing paediatric pain.

Response bias is a further potential limitation, not only for children being assessed, but also for anaesthetists who may change practice due to the presence of an auditor.

An analysis of pain categorised further into mild, moderate and severe; and associations with selected variables was included. In order to detect a medium effect size (if it exists), with $80 \%$ power, at the $5 \%$ significance level, a sample size of a minimum 108 patients was required. Only larger effect sizes (if they exist) could be detected with such a small sample size. This is a limitation in the study.

\section{Conclusion}

Audits are necessary to evaluate what resources are needed to optimise care. ${ }^{19}$ Despite the vast amount of literature available, perioperative pain management in patients presenting for tonsillectomy continues to be poorly managed. Appropriate premedication and two hours of clear liquid ingestion needs to be introduced. Where possible surgical technique should involve bipolar cautery and be limited to less than 45 minutes, and a preemptive, multimodal, opioid-sparing anaesthetic should be routinely practised.

\section{Acknowledgements}

This study was in fulfilment of the requirements for the degree of Master of Medicine in the branch of Anaesthesiology.

\section{Conflict of interest}

I declare that I have no financial or personal relationship(s) which may have inappropriately influenced me in compiling this paper.

\section{ORCID}

\section{PMogane (D) https://orcid.org/0000-0002-5523-4539}

\section{References}

1. Bolton $S$, Myles $P$, Nolan T, Sterne J. Prophylaxis of postoperative vomiting in children undergoing tonsillectomy: a systematic review and meta-analysis. British Journal of Anaesthesia. 2006;95(4):593-604.

2. Syed Ml, Magos TA, Singh J, Montague ML. A new analgesia regimen after (adeno) tonsillectomy in children: a pilot study. Clinical Otolaryngology. 2016;41(6):660-5.
3. Subramanyam R, Chidambaran V, Ding L, Myer C, Sadhasivam S. Anesthesia- and opioids-related malpractice claims following tonsillectomy in USA: LexisNexis claims database 1984-2012. Pediatric Anesthesia. 2014;24(4):412-20.

4. White MC, Nolan JA. An evaluation of pain and postoperative nausea and vomiting following the introduction of guidelines for tonsillectomy. Pediatric Anesthesia. 2005;15(8):683-8.

5. Pain terminology [Internet]. IASP Press. 1994 [cited 12 January 2016]. Available from: www.iasp-pain.org/Taxonomy.

6. Carr D, Goudas L. Acute pain. Lancet. 1999;353:2051-8.

7. Racine NM, Riddell RR, Khan M, Calic M, Taddio A, Tablon P. Systematic Review: Predisposing, Precipitating, Perpetuating, and Present Factors Predicting Anticipatory Distress to Painful Medical Procedures in Children. Journal of pediatric psychology. 2016;41(2):159-81.

8. Ramos SD, Mukerji S, Pine HS. Tonsillectomy and Adenoidectomy. Pediatric Clinics of North America. 2013;60(4):793-807.

9. Dadgarnia M, Aghaei M, Atighechi S, Bedniafard N, Vahidi M, Meybodian $M$, et al. The comparison of bleeding and pain after tonsillectomy in bipolar electrocautery vs cold dissection. International Journal of Pediatric Otorhinolaryngology. 2016;89:38-41.

10. Brand K, Thorpe B. Pain assessment in children. Anaesthesia and Intensive Care Medicine. 2016;17(6):270-3.

11. Ghai B, Makkar JK, Wig J. Postoperative pain assessment in preverbal children and children with cognitive impairment. Pediatric Anesthesia. 2008;18(6):462-77.

12. Royal College of Nursing. The recognition and assessment of acute pain in children. London: Royal College of Nursing; 2009.

13. Association of Paediatric Anaesthesia of Great Britain and Ireland. Good Practice in Postoperative and Procedural Pain Management. Pediatric Anesthesia. 2012;22:1-79.

14. Vlajkovic G, Sindjelic R. Emergence Delirium in Children: Many Questions, Few Answers. Anesthesia and Analgesia. 2007;104(1):84-91.

15. Dahmani S, Delivet $\mathrm{H}$, Hilly J. Emergence delirium in children: an update. Current Opinion in Anaesthesiology. 2014;27:309-15.

16. Sherwood S. Auditing paediatric pain management. Paediatric Nursing. 1998;10(6):15-7.

17. Shirley W, Wooley A, Wiatrak B. Pharyngitis and adenotonsilar disease. Cummins Otolaryngology - head and neck surgery. 3. Philadelphia: Mosby Elsevier; 2010. p. 2783-5, 95-96, 99-801.

18. Ikoma R, Sakane S, Niwa K, Kanetaka S, Kawano T, Oridate N. Risk factors for post-tonsillectomy hemorrhage. Auris Nasus Larynx. 2014;41(4):376-9.

19. British Association of Otorhinolaryngologists - Head and Neck Surgeons Comparitive Audit Group, Clinical Effectiveness Unit TRCoSoE. National prospective tonsillectomy audit: final report of an audit carried out in England and Northern Ireland between July 2003 and September 2004.May 200518 August 2016. Available from: http://www.entuk.org/members/audits/tonsils/ Tonsilectomyauditreport_pdf.

20. Pieters B, Penn E, Nichlaus P, Bruegger D, Mehta B, Weatherly R. Emergence delirium and postoperative pain in children undergoing tonsillectomy: a comparison of propofol vs sevoflurane anesthesia. Pediatric Anesthesia. 2010;20(10):944-50.

21. Wong-Baker FACES [Internet]. 2009-2015 [cited 15 January 2015].

22. Oztekin S, Hepaguslar H, Kar A, Ozzeybek D, Artikaslan O, Elar Z. Preemptive diclofenac reduces morphine use after remifentanil-based anaesthesia for tonsillectomy. Pediatric Anesthesia. 2002;12(8):694-9.

23. Tawalbeh $M$, Nawasreh 0 , Husban A. Comparative study of diclofenac sodium and paracetamol for treatment of pain after adenotonsillectomy in children. Saudi Medical Journal. 2001;22(2):121-3.

24. Schnabel A, Reichl S, Meyer-Friebem C, Zahn P, Pogatzki-Zahn E. Tramadol for postoperative pain in children Cochrane Database of Systematic Reviews: John Wiley and Sons; 2015 [updated 11 Novemebr 2016]. 18 March 2015:[1-72].

25. Lauder G, Emmott A. Confronting the challenges of effective pain management in children following tonsillectomy. International Journal of Pediatric Otorhinolaryngology. 2014;78(11):1813-27.

26. American Society of Anesthesiologists. Practice Guidelines for Preoperative Fasting and the Use of Pharmacologic Agents to Reduce the Risk of Pulmonary Aspiration: Application fo Healthy Patients Undergoing Elective Procedures. Anesthesiology 2011;114(3):495-511.

27. Elgueta MF, Echevarría GC, De la Fuente N, Cabrera F, Valderrama A, Cabezón $R$ et al. Effect of intravenous fluid therapy on postoperative vomiting in children undergoing tonsillectomy. British Journal of Anaesthesia. 2013;110(4):607-14.

28. Cote C, Lerman J, Todres D. A Practice of Anesthesia for Infants and Children. 4th edition ed. Philadelphia: Saunders Elsevier Health Sciences; 2009. 1167 p. 